\title{
Power of tests for quantitative trait loci detection using full-sib families in different schemes
}

\author{
HÉLÈNE MURANTY \\ Station d'Amélioration des Arbres Forestiers, INRA Centre d'Orléans, 45160 Ardon, France
}

\begin{abstract}
Strategies for mapping quantitative trait (QT) loci (QTL) need special studies in species where inbred lines cannot easily be obtained. We propose the detection of QTL with schemes including several full-sib families and study here the efficiency of such schemes - diallel, factorial, cyclic or single-pair mating designs - for a varying number of parents, on the basis of the power of tests for QTL detection. We considered a single, fully informative marker completely linked to the QTL. The tests proposed for QTL detection are based on linear additive models. The results show that for a given number of parents, in given conditions, the mating design has no effect on power. It increases as the total number of individuals genotyped increases. The number of parents affects the power of QTL detection insofar as the effect of substituting QT alleles varies from one parent to another and as some parents can be homozygous at the QTL: with only two parents QTL detection is often less powerful than with more parents. The size of the genetic effects that can be detected with a given power decreases as the number of parents increases from two to six and is quite stable for more than six parents. If QTL heterozygote frequency in the base population is high enough (greater than 0.2) mating designs with six parents should give a good sample of variance attributable to QTL and allow the detection of QTL with reasonable power.
\end{abstract}

Keywords: diallel cross, full-sib families, molecular markers, power of test, QTL detection.

\section{Introduction}

Quantitative phenotypic traits in many living creatures show continuous variation as a result of environmental and multiple gene actions. These genes are located at loci called quantitative trait (QT) loci (QTL) that generally cannot be identified or mapped on the basis of quantitative variation only. An indirect approach to the problem was proposed by Sax (1923): QTL can be detected when they cosegregate with marker loci.

The first QTL mapping experiments using linkage maps were performed on F2 or back-cross (BC) populations derived from inbred lines, in tomato and maize (e.g. Tanksley et al., 1982; Edwards et al., 1987) and then in other species where inbred lines are easy to obtain [barley (Barua et al., 1993), rice (Wang et al., 1994) and soybean (Concibido et al., 1994)]. Strategies for QTL mapping in populations derived from inbred lines have been studied theoretically by many authors (Soller et al., 1976; Weller, 1986; Lander \& Botstein, 1989; Soller \& Beckmann, 1990; Luo \& Kearsey, 1991; Haley \& Knott, 1992;
Martinez \& Curnow, 1992; Kearsey \& Hyne, 1994). These strategies must be adapted, however, for species where inbred lines are difficult to obtain such as animal species, fruit and forest trees or selfincompatible plant species. Two types of strategy have been proposed so far: $F_{2}$-like strategies and $\mathrm{BC}$-like strategies. In the former, the population studied is an $F_{2}$ segregating family produced by the cross of two full-sib individuals. The $F_{0}$ parents are chosen to be very different for the phenotypic trait studied so that they can be supposed to be homozygous at the QTL, with different alleles (Beckmann \& Soller, 1988; Knott \& Haley, 1992; Williams \& Neale, 1992; Haley et al., 1994). In the latter, segregating families come from parents supposed to be heterozygous at the QTL. The families can be one or more half-sibs (daughter and grand-daughter designs; Weller, 1990; Williams \& Neale, 1992) or a full-sib family (double pseudo test cross; LeonardsSchippers et al., 1994) or many independent full-sib families coming from a random mating population (Soller \& Genizi, 1978; Knott, 1994) or full-sib 
families related in a hierarchical structure (Götz \& Ollivier, 1992).

Mating designs like diallel or factorial designs are suitable for estimating genetic parameters such as additive and dominance variance and heritabilities. At the beginning of forest tree improvement programmes, they are often used for this purpose (Stonecypher \& McCullough, 1986; Pichot \& Teissier du Cros, 1989). In many species, they are also used to select the genotypes that will be crossed to produce improved populations, on the basis of their general combining ability (gca). Cyclic and singlepair mating designs are used to produce recombined genotypes that candidate for selection. If one plans to use full-sib families produced in a mating design to detect and map QTL, should one use one family or more? If the answer is several families, should they be independent or related? These questions can be answered on the basis of the power of tests for QTL detection.

Statistical methods used to detect QTL generally suppose that QTL act on the phenotypic trait mean. In the simpler approach (see e.g. Soller et al., 1976), marker genotypes are considered as factors in a one-way ANOVA, and the test for significance of this factor is the test for the presence of a QTL in the vicinity of the marker locus. This method, however, gives no information on the real position of the QTL and confuses its quantitative effects with its distance from the marker. More elaborate methods using flanking markers, either with a log-likelihood approach (Lander \& Botstein, 1989) or a multiple regression approach (Knapp, 1991; Haley \& Knott, 1992; Martinez \& Curnow, 1992; Jansen, 1993; Moreno-Gonzalez, 1993; Rodolphe \& Lefort, 1993; Zeng, 1994) allow separate estimation of QTL positions and effects. They have a greater power, particularly when the molecular map is sparse (Rebaï et al., 1995). Moreover, in cases of tightly linked QTL, the multiple regression approach using more than the flanking markers should enable the avoidance of false localization and have a greater power than ANOVA or methods using only the flanking markers. However, the different structures of segregating populations should affect power in the same way whatever the methodology of QTL detection used. Consequently, power can be calculated assuming that ANOVA tests will be performed.

The purpose of this paper is to compare the power of tests for QTL detection given different structures of segregating populations: different mating designs (diallel designs, without selfing or reciprocals, and factorial, cyclic and single-pair designs), with varying numbers of parents.

\section{Theory}

\section{Mating designs studied}

In a diallel mating design without selfing or reciprocals with $p$ parents, each parent is crossed with the $p-1$ other parents and serves as male or female. This design is used with hermaphrodite plants to estimate the performance of the genotype as a parent in general $(g c a)$ and to identify eventually pairs of parents performing better (or worse) than predicted by their $g c a$.

In a factorial mating design with $p_{1}$ and $p_{2}$ parents, each female of a set of $p_{1}$ females is crossed with each male of a set of $p_{2}$ males. This design is used with dioecious plants, with the same goals as a diallel mating design.

In a cyclic design with $p$ parents, each parent is crossed with only two other parents, and serves as a male or female so that the crosses form a chain: parent 1 is crossed with parent 2,2 with 3,3 with 4 , etc., and $p$ with 1 . In a single-pair design with $p$ parents, each parent is crossed with only one other parent so that $p / 2$ full-sib families are produced. The cyclic and single-pair designs are used essentially to produce individuals that are evaluated for themselves and give little information on the parents.

In diallel, factorial and cyclic designs, the full-sib families are related, when they share a common parent, whereas in single-pair designs they are unrelated, unless the parents are related.

\section{Genetic and statistical models}

To compare different mating designs, we held the total number of individuals $N$ constant. For each mating design, we assumed that the number of individuals was the same for each full-sib family. We supposed that the QTL was on the marker, meaning that the recombination rate between QTL and marker locus was zero. To facilitate statistical treatment, we assumed the marker was fully informative. When this assumption holds, in the offspring of a cross between parents $i\left(i_{1} i_{2}\right)$ and $j\left(j_{1} j_{2}\right)$ four genotypic classes are distinguishable: $i_{1} j_{1}, i_{1} j_{2}, i_{2} j_{1}$ and $i_{2} j_{2}$, segregating with a 1:1:1:1 ratio. We used a completely additive model, neglecting dominance (and epistasis) at QTL and between-family levels. The only estimable parameters at the QTL level are the differences between the two alleles of each parent. So we wrote:

$Y_{i_{a} j_{b} k}=\mu+g c a_{i}+g c a_{j}+x_{i_{a}} \cdot q_{i}+x_{j_{b}} \cdot q_{j}+\varepsilon_{i_{a} j_{b} k}$

where $Y_{i_{a} j_{b} k}$ is the phenotype of the $k$ th individual of genotypic class $i_{a} j_{b}, \mu$ is the general mean, $g c a_{i}$ is the 
gca of parent $i, 2 q_{i}$ is the effect of substituting one allele of parent $i$ with the other, $x_{i_{a}}$ is a dummy variable which takes a value of 1 for plants of genotype $i_{1} j_{1}$ or $i_{1} j_{2}$ and of -1 for plants of genotype $i_{2} j_{1}$ or $i_{2} j_{2}$, and $\varepsilon_{i_{a} j_{b} k}$ is the residual error term with variance $\sigma^{2}$. The $g c a_{i}$ effect of parent $i$ is the sum of the means of the effects of its alleles at all QTL affecting the trait studied. The error comprises the effects of other segregating QTL and an environmental effect. It should be noted that the QTL effect is nested in the gca effect.

\section{Matrix notation}

Classical constraints were applied to the gca parameters, depending upon the mating design studied (see Table 1), to determine the models. With a matrix notation, they can be written as follows: $\mathbf{Y}=\mathbf{Z} \boldsymbol{\beta}+\varepsilon$, where $\mathbf{Y}$ is the $N \times 1$ vector of phenotypes, $\mathbf{Z}$ is the $N \times k$ design matrix (of rank $k$ ) taking into account the constraints, $\boldsymbol{\beta}$ is the $k \times 1$ vector of free parameters and $\varepsilon$ is a $N \times 1$ random vector of residuals with mean 0 and variance $\sigma^{2} \mathbf{I}$ (I is the identity matrix). The $\mathbf{Z}$ matrix and $\boldsymbol{\beta}$ vector can be partitioned into three parts: $\mathbf{Z}=\left[\mathbf{Z}_{0}\left|\mathbf{Z}_{1}\right| \mathbf{Z}_{2}\right]$ and $\boldsymbol{\beta}^{\prime}=\left[\boldsymbol{\beta}_{0}^{\prime}\left|\boldsymbol{\beta}_{1}^{\prime}\right| \boldsymbol{\beta}_{2}^{\prime}\right]$ where $\boldsymbol{\beta}_{0}^{\prime}=[\mu], \boldsymbol{\beta}_{1}$ corresponds to the free parameters representing gca or family effects and $\boldsymbol{\beta}_{2}^{\prime}=\left[q_{i}\right]_{i=1, \ldots, p} \quad(p$ is the number of parents and a prime indicates the transpose of a matrix or a vector).

\section{Hypothesis testing}

To test for the presence of a QTL linked to the marker, we have to test the hypothesis $\mathrm{H}_{0}: \forall i, q_{i}=0$ against $\mathrm{H}_{1}: \exists i / q_{i} \neq 0$. The $\mathrm{H}_{0}$ hypothesis can be written with the matrix notation as $\mathbf{K} \boldsymbol{\beta}=[0]$ and $\mathbf{K}=[0|0| \mathbf{I}]$. For this test, the statistic to consider is the classical $F$ statistic, which can be written as

$W=\frac{\mathbf{Y}^{\prime}\left(\mathbf{Z Z}^{-}-\left[\mathbf{Z}_{0} \mid \mathbf{Z}_{1}\right]\left[\mathbf{Z}_{0} \mid \mathbf{Z}_{1}\right]^{-}\right) \mathbf{Y}}{\mathbf{Y}^{\prime}\left(\mathbf{I}-\mathbf{Z} \mathbf{Z}^{-}\right) \mathbf{Y}} \frac{N-k}{p}$

where $\mathbf{Z}^{-}=\left(\mathbf{Z}^{\prime} \mathbf{Z}\right)^{-1} \mathbf{Z}^{\prime}$ (Graybill, 1976, p. 194). Under $\mathrm{H}_{0}, W$ follows a central $F$ distribution with $(p$, $N-k)$ d.f., whereas under $\mathrm{H}_{1}$ it is distributed as a non-central $F$ random variable with $(p, N-k)$ d.f. and a non-centrality parameter $\lambda . \mathrm{H}_{0}$ is rejected if $W \geq F_{\alpha(p, N-k)}$. The non-centrality parameter can be calculated from the following formula:

$\lambda=\frac{(\mathbf{K} \boldsymbol{\beta})\left[\mathbf{K}\left(\mathbf{Z}^{\prime} \mathbf{Z}\right)^{-1} \mathbf{K}^{\prime}\right]^{-1}(\mathbf{K} \boldsymbol{\beta})}{2 \sigma^{2}}$

(Graybill, 1976).

\section{Power calculations}

The power of a test is defined as the probability of rejecting the null hypothesis when its alternative is true. In our case, the power is the probability of detecting a QTL near the marker when it exists. An expression for the power of the test is

$$
\begin{aligned}
P & =\operatorname{Pr}\left(F^{\prime}(p, N-k, \lambda)>F_{\alpha}(p, N-k)\right) \\
& =\int_{F_{x(0, N-k)}}^{+\infty} F_{x}^{\prime}(p, N-k, \lambda) d x .
\end{aligned}
$$

So the power is a function of the total number of individuals studied, $N$, the number of parents, $p$, the significance level, $\alpha$ chosen, and the non-centrality parameter $\lambda$, which depends on the mating design. With algebraic calculations, the $\lambda$ parameter could be expressed as a function of $N, \sigma^{2}$ and $\boldsymbol{\beta}_{2}$ for each mating design studied (see Appendix and Table 1). For these calculations, we assumed that all genotypic classes in a family have the same number of individuals.

Before any experience, QTL effects are unknown. We fixed the variance attributable to QTL effects in the base population, $\sigma_{\mathrm{QTL}}^{2}$, to 10 and let $\sigma^{2}$ vary to simulate QTL which explain a variable part of the phenotypic variance. To express the results, we will consider the more classical parameter 'explained phenotypic variance' (EPV) which is $\sigma_{\mathrm{QTV}}^{2} /\left(\sigma_{\mathrm{QTL}}^{2}+\sigma^{2}\right)$ : this is in fact the within-family phenotypic variance explained by QTL effects. To choose the values of $q_{i}$ we considered that they are related to $\sigma_{\mathrm{QTL}}^{2}$ by the formula

$\sigma_{\mathrm{QTL}}^{2}=\sum_{i=1}^{N p} q_{i}^{2} / N p$,

where $i$ is any parent of the base population and $N p$ is the size of this population. We looked at two situations: biallelic and multiallelic QTL.

When the QTL is biallelic, only the parents heterozygous at the QTL have non-zero QTL effects and all of these parents have the same QTL effect, $q$. The variance attributable to QTL effects in the base population can then be written as $\sigma_{\mathrm{OTL}}^{2}=\mathrm{He} \times q^{2}$, where $\mathrm{He}$ is the heterozygote frequency. So, in power calculations, $q$ was replaced by $\pm \sqrt{10 / H e}$. Moreover, power depends on $H$, the number of parents heterozygous at the QTL involved in the mating designs, and it can be calculated as

$$
\sum_{h=0}^{p} \operatorname{Pr}(H=h) \times P_{H=h}
$$

where $\operatorname{Pr}(H=h)$ is the probability of having $h$ parents heterozygous at the QTL involved in the 


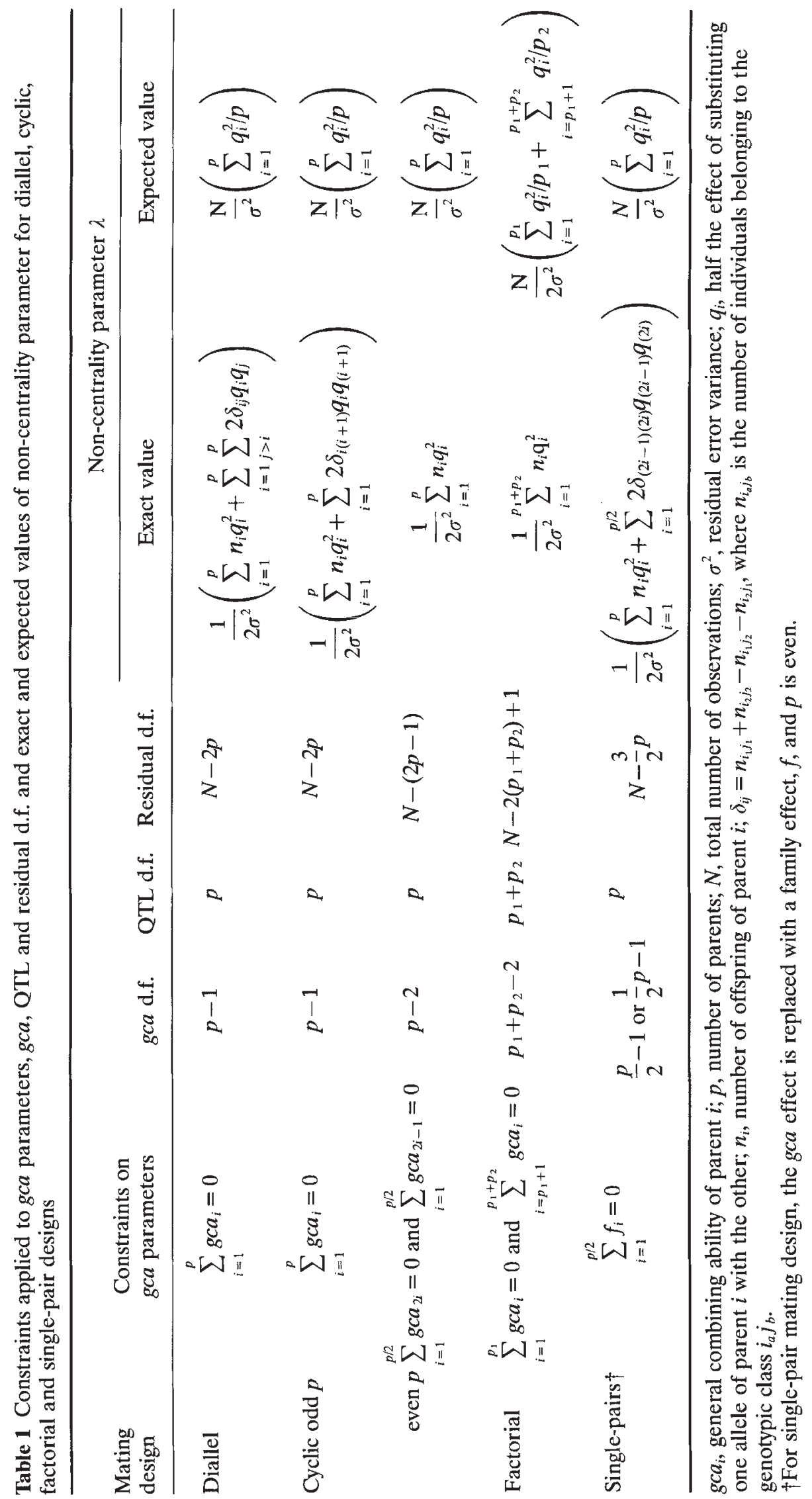

(c) The Genetical Society of Great Britain, Heredity, 76, 156-165. 
mating designs and $P_{H=h}$ is the power obtained when $H=h . H$ is a random binomial variable with parameters $p$ (number of parents in the mating design) and $\mathrm{He}$.

When the QTL is multiallelic, parents homozygous at the QTL are rarer, and different heterozygous parents have different QTL effects. In this case, QTL effects were simulated using a normal distribution with mean 0 and variance 10 .

We developed FORTRAN 77 programs using NAG routines (Numerical Algorithms Group, 1992) to perform all the calculations.

\section{Results}

Table 1 shows that for all mating designs, the non-centrality parameter $\lambda$ could be expressed as

$$
\frac{N}{\sigma^{2}} \times \hat{\sigma}_{\mathrm{QTL}}^{2}
$$

where $\hat{\sigma}_{\text {OTL }}^{2}$ is an estimate of the variance attributable to QTL effects. It depends essentially on the number of parents, $p$, and little on the mating design. As a result, when $N$ (total number of individuals observed and genotyped), $\sigma^{2}$ (residual variance) and $\alpha$ (significance level) are fixed, curves representing power as a function of $p$ for different mating designs are indistinguishable (data not shown).

Powers were calculated for different values of $p$, $N, \sigma^{2}$ and $\alpha$. To take into account the fact that tests are performed all along the genome, the significance level per test should be set at between approximately 1 per cent and 0.1 per cent depending upon the density of the map (Rebai et al., 1995). However, the influence of $p$ and $E P V$ on power are similar whatever the significance level chosen, so we present results obtained with $\alpha$ fixed to 1 per cent.

\section{Biallelic QTL}

Table 2 presents the power of QTL detection as a function of $p, E P V, N$ and $H e$ when a biallelic QTL is considered. If $p$ is fixed, the power increases as $E P V$ and/or $N$ increase, which is usual, and tends to a limit. This limit is $\operatorname{Pr}(H>0)$ : in fact, when $E P V$ and $N$ are high enough, power conditional on $H$ (the number of heterozygous parents effectively involved in the crosses) is 100 per cent unless $H=0$, in which case power is null. The limit $\operatorname{Pr}(H>0)$ increases when $p$ and/or $\mathrm{He}$ increase. But when $\mathrm{He}=0.2$, the limit, and consequently the power when $E P V$ and $N$ are high, increase markedly and regularly as $p$ increases, whereas when $\mathrm{He}=0.5$, the limit increases by 25 per cent from two to 12 parents, but is quite similar with four, six or 12 parents.

We calculated the minimum genetic effect sizes detectable with 90 per cent power when $N$ is fixed to 300. When $H e=0.2,90$ per cent power is reached only with 11 parents or more. With six parents for example, the maximum power is about 75 per cent (Table 2). When $\mathrm{He}=0.5,90$ per cent power is reached only with four parents or more, and the minimum genetic effect detected with this power is the same whatever the number of parents, e.g. 13 per cent with four parents, 11 per cent with five, 10 per cent with nine and 12 per cent with 14 parents. We investigated the influence of $N$ on the size of the minimum detectable genetic effect with 90 per cent power, with $p$ fixed at 6 and $\mathrm{He}$ fixed at 0.5 . From 100 to 300 individuals, it decreased sharply, from 29 per cent to 10 per cent; it then fell more slowly, reaching 4.1 per cent with 1000 individuals, and decreasing only very slowly when $N$ increased above 1000. We also calculated the population size required to detect an effect explaining 1 per cent of within-family phenotypic variance with a significance level of 5 per cent and a power of 90 per cent: for six parents, the population size is $\sim 2500$; for between six to 18 parents it does not vary much, and increases slightly for more than 20 parents.

\section{Multial/elic OTL}

Table 3 presents the power of QTL detection as a function of $p, E P V$ and $N$ when a multiallelic QTL is considered. Again, if $p$ is fixed, the power increases with $E P V$ and/or $N$, but it always tends to 100 per cent. Moreover, $p$ has a minor effect on power; the highest variations in power caused by $p$, when $E P V$ and $N$ are fixed, are about 40 per cent, ranging from 10 to 50 per cent. The effect of $p$ on power results from variation in the estimation of $E P V$ with the parents sampled because all potential parents are heterozygous at the QTL, though with variable QTL effects. $p$ also influences the minimum genetic effect sizes detectable with 90 per cent power when $N$ is fixed at 300: this effect decreases from 24 per cent with two parents to 15 per cent with four parents and is quite stable with more than that, e.g. 14 per cent with five, 13 per cent with nine and 14 per cent with 14 parents.

\section{Discussion}

We considered a fully informative marker, giving rise to four genotypic classes in each family. If any 
Table 2 Power to detect a biallelic QTL as a function of number of parents $p$, number of individuals $N$, heterozygosity $H e$ and explained phenotypic variance $E P V$

\begin{tabular}{|c|c|c|c|c|c|c|c|c|c|c|c|c|c|c|}
\hline \multirow{3}{*}{$\begin{array}{l}\mathrm{He} \\
N \\
E P V\end{array}$} & \multicolumn{14}{|c|}{0.2} \\
\hline & \multicolumn{7}{|c|}{100} & \multicolumn{7}{|c|}{500} \\
\hline & 0.5 & 1 & 2 & 5 & 10 & 20 & 50 & 0.5 & 1 & 2 & 5 & 10 & 20 & 50 \\
\hline \multicolumn{15}{|l|}{$p$} \\
\hline 2 & 3 & 5 & 11 & 28 & 36 & 37 & 37 & 15 & 28 & 36 & 37 & 37 & 37 & 37 \\
\hline 3 & 2 & 4 & 9 & 26 & 44 & 49 & 49 & 12 & 26 & 43 & 49 & 49 & 49 & 49 \\
\hline 4 & 2 & 3 & 7 & 22 & 44 & 59 & 59 & 10 & 23 & 44 & 59 & 59 & 59 & 59 \\
\hline 5 & 2 & 3 & 6 & 20 & 42 & 64 & 68 & 8 & 20 & 42 & 66 & 68 & 68 & 68 \\
\hline 6 & 2 & 2 & 5 & 17 & 40 & 66 & 74 & 7 & 18 & 40 & 69 & 74 & 74 & 74 \\
\hline 8 & 1 & 2 & 4 & 14 & 35 & 66 & 83 & 6 & 15 & 36 & 70 & 83 & 83 & 83 \\
\hline 10 & 1 & 2 & 3 & 11 & 31 & 65 & 89 & 5 & 12 & 32 & 70 & 87 & 89 & 89 \\
\hline 12 & 1 & 2 & 3 & 9 & 27 & 62 & 92 & 4 & 11 & 29 & 70 & 88 & 93 & 93 \\
\hline 14 & 1 & 2 & 3 & 8 & 24 & 60 & 94 & 4 & 9 & 26 & 69 & 89 & 95 & 96 \\
\hline
\end{tabular}

$\mathrm{He}$

0.5

\begin{tabular}{|c|c|c|c|c|c|c|c|c|c|c|c|c|c|c|}
\hline \multirow{2}{*}{$\begin{array}{l}N \\
E P V\end{array}$} & \multicolumn{7}{|c|}{100} & \multicolumn{7}{|c|}{500} \\
\hline & 0.5 & 1 & 2 & 5 & 10 & 20 & 50 & 0.5 & 1 & 2 & 5 & 10 & 20 & 50 \\
\hline
\end{tabular}

\begin{tabular}{|c|c|c|c|c|c|c|c|c|c|c|c|c|c|c|}
\hline \multicolumn{15}{|l|}{$p$} \\
\hline \multicolumn{15}{|l|}{$p_{2}$} \\
\hline 3 & 2 & 3 & 7 & 24 & 53 & 80 & 88 & 10 & 24 & 51 & 82 & 88 & 88 & 88 \\
\hline 4 & 2 & 3 & 6 & 20 & 49 & 81 & 94 & 8 & 20 & 47 & 84 & 93 & 94 & 94 \\
\hline 5 & 2 & 3 & 5 & 17 & 45 & 81 & 97 & 7 & 18 & 44 & 84 & 95 & 97 & 97 \\
\hline 6 & 2 & 2 & 4 & 15 & 41 & 80 & 98 & 6 & 16 & 41 & 84 & 96 & 98 & 98 \\
\hline 8 & 1 & 2 & 4 & 12 & 35 & 77 & 99 & 5 & 13 & 35 & 83 & 97 & 99 & 100 \\
\hline 10 & 1 & 2 & 3 & 10 & 30 & 73 & 99 & 4 & 11 & 31 & 81 & 98 & 100 & 100 \\
\hline 12 & 1 & 2 & 3 & 8 & 26 & 69 & 100 & 4 & 10 & 28 & 79 & 98 & 100 & 100 \\
\hline 14 & 1 & 2 & 3 & 7 & 22 & 65 & 100 & 4 & 8 & 25 & 77 & 98 & 100 & 100 \\
\hline
\end{tabular}

classes were confused, then power would decrease. Many alleles are necessary to obtain a fully informative marker for crosses between several parents. It is impossible for isoenzymes or restriction fragment length polymorphisms to give many alleles regularly but this may be only a technical limit when the parents chosen are unrelated and not inbred. Microsatellite techniques could give enough alleles, but at higher cost. On the other hand, the use of all of the markers in a linkage group should make it possible to estimate the probabilities, for each individual, of belonging to each of the four genotypic classes of its family at each point of a chromosome. Haley et al. (1994) showed that in an outbred pedigree with a flanking marker method, this approach can partly overcome the loss of power because of partially informative markers. It should also be true for schemes like the ones we are studying here.
Calculating the non-centrality parameter $\lambda$ we replaced the number of individuals per genotypic class by its expectation under the assumption that all families and all genotypic classes in a family contain the same number of individuals. Because the QTL effect tested is nested in the gca or family effect, for a given number of parents under the given conditions the different mating designs studied are equivalent. So, in planning QTL mapping experiments one should choose mating designs that give enough individuals per family to estimate recombination rates between markers accurately. This should produce at least one individual per genotypic class. Moreover, with some simulations, we verified that the fluctuations in the number of individuals per genotypic class (with a constant number of individuals per family) have little consequences on power (data not shown). 
Table 3 Power to detect a multiallelic QTL as a function of number of parents $p$, number of individuals genotyped $N$, and explained phenotypic variance $E P V$

\begin{tabular}{|c|c|c|c|c|c|c|c|c|c|c|c|c|c|c|}
\hline \multirow{2}{*}{$\begin{array}{l}N \\
E P V\end{array}$} & \multicolumn{7}{|c|}{100} & \multicolumn{7}{|c|}{500} \\
\hline & 0.5 & 1 & 2 & 5 & 10 & 20 & 50 & 0.5 & 1 & 2 & 5 & 10 & 20 & 50 \\
\hline \multicolumn{15}{|l|}{$p$} \\
\hline 2 & 2 & 5 & 10 & 28 & 47 & 70 & 91 & 13 & 27 & 47 & 71 & 85 & 93 & 98 \\
\hline 3 & 2 & 4 & 7 & 24 & 48 & 72 & 94 & 10 & 24 & 47 & 78 & 90 & 97 & 100 \\
\hline 4 & 2 & 3 & 6 & 20 & 45 & 76 & 97 & 9 & 21 & 45 & 79 & 93 & 98 & 100 \\
\hline 5 & 2 & 3 & 5 & 17 & 42 & 75 & 98 & 7 & 19 & 42 & 79 & 94 & 99 & 100 \\
\hline 6 & 2 & 2 & 5 & 16 & 39 & 74 & 99 & 7 & 16 & 39 & 79 & 95 & 99 & 100 \\
\hline 8 & 1 & 2 & 4 & 13 & 35 & 72 & 99 & 5 & 13 & 36 & 78 & 95 & 100 & 100 \\
\hline 9 & 1 & 2 & 3 & 11 & 32 & 71 & 99 & 5 & 12 & 33 & 77 & 96 & 100 & 100 \\
\hline 10 & 1 & 2 & 3 & 10 & 30 & 70 & 99 & 4 & 12 & 31 & 76 & 96 & 100 & 100 \\
\hline 12 & 1 & 2 & 3 & 9 & 26 & 66 & 99 & 4 & 10 & 29 & 75 & 97 & 100 & 100 \\
\hline 14 & 1 & 2 & 3 & 7 & 23 & 63 & 99 & 4 & 9 & 25 & 74 & 96 & 100 & 100 \\
\hline
\end{tabular}

We supposed that the recombination rate between the QTL and marker locus was zero. As stated by Soller et al. (1976) when the QTL and marker locus are not confounded, genetic effects $\left(q_{i}\right.$ here) are multiplied by a factor of $1-2 r$, where $r$ is the recombination rate between the QTL and the marker locus. Consequently the power decreases as $r$ increases. This problem can be partially overcome with interval mapping methods. To avoid a large loss of power even with interval mapping methods, the molecular map should be sufficiently dense, with one marker approximately every $20-30 \mathrm{cM}$.

In our statistical model we introduced only additive effects at the QTL and between-family levels. Dominance effects could be added at the betweenfamily levels if dominance is known to exist: this would reduce within-family variance and consequently increase power. At the QTL level, adding dominance parameters would lead to complicated calculations. Rebaï \& Goffinet (1993) demonstrated that at the QTL detection step, it is better to neglect dominance if it is not very large. Moreover, de Vienne et al. (1994) suggested that dominance and epistasis can be very important at the molecular level, i.e. for quantitative traits like mRNA or protein amounts, but with integration at higher levels, i.e. for macroscopic traits whose values vary in direct ratio to the metabolic flux, as more and more genes have an effect, dominance and epistasis become negligible. Consequently, for quantitative traits considering an organism as a whole, QTL effects will be mainly additive. All this justifies neglecting dominance (and epistasis) at the QTL level.

Power calculations were made assuming two types of QTL, biallelic and multiallelic. So what type of QTL can be assumed? Evidence of the existence of more than two QT alleles has been recently reported in the diploid potato (Van Eck et al., 1994) and loblolly pine (Groover et al., 1994). However, an infinite number of QT alleles, like those we simulated for multiallelic QTL, is impossible; the real case is between the two models of QTL studied. The number of QT alleles is probably low if the population the parents come from was founded with a limited number of genotypes and/or underwent endogamy for many generations. This corresponds roughly to races of domestic animal species and fruit tree species. On the contrary, the number of QT alleles is probably large in natural populations if they are selectively neutral. Forest tree breeding populations, recently founded from many genotypes, could reveal many QT alleles. Anyway, the levels of power obtained with the two models are similar under the given conditions, except when the power reaches its limit in the case of a biallelic QTL. So our conclusions should be valid for any type of QTL: for two, three, or many alleles. For a biallelic QTL, the power is limited clearly under 100 per cent when the probability that all parents chosen are homozygous at the QTL is not negligible. For a given trait, this situation could occur on one, but not all QTL.

A first result of this study is that one of the parameters determining the power of QTL detection is the number of parents in the segregating population and not the number of families, which was the parameter that was investigated by Soller \& Genizi (1978); e.g. with six parents, the same efficiency is obtained with diallel (15 full-sib families) and single- 
pair (three families) mating designs. A diallel (or factorial) design should still be used to allow the estimation of dominance components at the QTL level and to prevent confusion of additive and dominance effects.

With the two types of QTL considered, the general trends are similar. Power increases when the variance explained by QTL and/or the population size increase, and when these factors determine a low power level, the power decreases as the number of parents increases. However, at a high power level, it increases as the number of parents increases. Similar results were found by Weller et al. (1990) for half-sib designs. As a consequence, the use of only one full-sib family for QTL detection is often less powerful than the use of mating designs with more parents, especially when QTL effects to be detected explain more than 10 per cent of phenotypic variance. This result is obtained because we considered that the parents, which have different QTL genotypes, are randomly chosen, with no account taken of the expected segregation of QTL in the progenies - it can be unknown. Hence, the QTL effects that effectively determine power are a sample of the QTL variance in the whole base population, to which we referred in explained phenotypic variance. The variance attributable to QTL is better sampled with more than two parents than with only two parents. As far as we know, experimental schemes aimed at detecting QTL in non-inbred plant species using full-sib families used only one family (Groover et al., 1994; Leonards-Schippers et al., 1994). Groover et al. (1994) used an $\mathrm{F}_{2}$-like strategy to obtain an efficient scheme. We demonstrate with this study that it is generally more prudent to divide the means among several families, involving at least four to six parents.

In a full-sib family design, each individual is used to estimate two contrasts, that between the alleles of its mother and that between the alleles of its father, whereas in a half-sib family design, each individual is used to estimate only one contrast, between the alleles of the common parent. Weller et al. (1990) showed that an effect of about 5 per cent would be detected with 50 per cent power with a type I error of 1 per cent when 1000 individuals from five halfsib families are genotyped. With the same parameters, we found that the power is 90 per cent. Consequently, whenever it is possible, full-sib family designs are preferable to half-sib family designs for the purpose of mapping QTL.

We saw that the minimum size of genetic effect detectable with 90 per cent power was quite large (15 per cent) for a reasonable size of population (about 300 individuals). Only six QTL of this size would explain 90 per cent of phenotypic variance. One solution to detect smaller QTL effects is to increase the population size. For example, the population size required to detect a 1 per cent genetic effect with 90 per cent power, when the type I error is 5 per cent, is quite high (3000) but is much lower than the population size obtained for the same constraints for independent full-sib families by Soller \& Genizi (1978), which was about 10000 . This is because they considered a two-allelic marker so that a heterozygous offspring of two heterozygous parents gave no information on QTL genotype and was discarded. We considered here a multiallelic marker that is always fully informative. Götz \& Ollivier (1992) and Knott \& Haley (1992) also showed that the use of fully informative markers would greatly increase the power of QTL detection. A complementary solution to detect smaller QTL effects is to control the genetic part of residual variation with multiple regression.

All the data in this paper give an idea of the means one has to invest to be able to detect a QTL of a given size. However, the gain in power that can be obtained with interval mapping when the recombination fractions between the QTL and marker is not zero should be further studied to give more precise indications. How to use the nearer informative markers to estimate probable genotypes of individuals also needs further investigation.

\section{Acknowledgements}

Thanks are due to the referees whose comments led to a far better presentation.

\section{References}

BARUA, U. M., CHALMERS, K. J., THOMAS, W. T. B., HACKETT, C. A., LEA, V., JACK, P., FORSTER, B. P., WAUGH, R. AND POWELL, W. 1993. Molecular mapping of genes determining height, time to heading, and growth habit in barley. Genome, 36, 1080-1087.

BECKMANN, J. S. AND SOLLER, M. 1988. Detection of linkage between marker loci and loci affecting quantitative traits in crosses between segregating populations. Theor. Appl. Genet., 76, 228-236.

CONCIBIDO, v. C., DENNY, R. L., BOUTIN, S. R., HAUTEA, R., ORF, J. H. AND YOUNG, N. D. 1994. DNA marker analysis of loci underlying resistance to soybean cyst nematode Heterodera glycines Ichinohe. Crop Sci., 34, 240-246.

DE VIENne, D., Josse, J. M., MAUrice, A., CAUSSE, M., LEONARDI, A., TOUZET, P., KREJCI, E., GOUESNARD, B., SANOU, J., PANOUILle, A., BOYAT, V., DUBREUIL, V., DUfour, P., GAllais, A., LEForT, M., ChaRCoSSET, A. AND DAMERVAL, C. 1994. Marquage et expression du 
génome chez le maïs. Génét. Sél. Évol. 26, Suppl. 1, 21-34.

EDWARDS, M. D., STUBER, C. W. AND WENDEL, J. F. 1987. Molecular-marker-facilitated investigations of quantitative-trait loci in maize. I. Numbers, genomic distribution and types of gene action. Genetics, 116, 113-125.

GÖTZ, K. U. AND OLLIVIER, L. 1992. Theoretical aspects of applying sib-pair linkage tests to livestock species. Génét. Sél. Évol., 24, 29-42.

GRAYBILL, F. A. 1976. Theory and Application of the Linear Model. Wadsworth, Belmont, California.

GROOVER, A., DEVEY, M., FIDDLER, T., LEE, J., MEGRAW, R., MITCHEL-OLDS, T., SHERMAN, B., VUJCIC, S., WILLIAMS, C. AND NEALE, D. 1994. Identification of quantitative trait loci influencing wood specific gravity in an outbred pedigree of loblolly pine. Genetics, 138, 1293-1300.

HALEY, C. S. AND KNOTT, S. A. 1992. A simple regression method for mapping quantitative trait loci in line crosses using flanking markers. Heredity, 69, 315-324.

HALEY, C. S., KNOTT, S. A. AND ELSEN, J. M. 1994. Mapping quantitative trait loci in crosses between outbred lines using least squares. Genetics, 136, 1195-1207.

JANSEN, R. C. 1993. Interval mapping of multiple quantitative trait loci. Genetics, 135, 205-211.

KEARSEY, M. J. AND HYNE, v. 1994. QTL analysis: a simple 'marker-regression' approach. Theor. Appl. Genet., 89, 698-702.

KNAPP, s. J. 1991. Using molecular markers to map multiple quantitative trait loci: models for backcross, recombinant inbred, and doubled haploid progeny. Theor. Appl. Genet., 81, 333-338.

KNOTT, S. A. 1994. Prediction of the power of detection of marker-quantitative trait locus linkages using analyses of variance. Theor. Appl. Genet., 89, 318-322.

KNOTT, S. A. AND HALEY, c. s. 1992. Maximum likelihood mapping of quantitative trait loci using full-sib families. Genetics, 132, 1211-1222.

LANDER, E. S. AND BOTSTEIN, D. 1989. Mapping Mendelian factors underlying quantitative traits using RFLP linkage maps. Genetics, 121, 185-199.

LEONARDS-SCHIPPERS, C., GIEFFERS, W., SHAFER-PREGL, R., RITTER, E., KNAPP, S. J., SALAMINI, F. AND GEBHARDT, C. 1994. Quantitative resistance to Phytophtora infestans in potato: a case study for QTL mapping in an allogamous plant species. Genetics, 137, 67-77.

LUO, Z. W. AND KEARSEY, M. J. 1991. Maximum-likelihood estimation of linkage between a marker gene and a quantitative trait locus. II. Application to backcross and doubled haploid populations. Heredity, 66, 117-124.

MARTINEZ, A. AND CURNOW, R. N. 1992. Estimating the locations and the sizes of the effects of quantitative trait loci using flanking markers. Theor. Appl. Genet., 85, 480-488.

MORENO-GONZALEZ, J. 1993. Efficiency of generations for estimating marker-associated QTL effects by multiple regression. Genetics, 135, 223-231.

NUMERICAL ALGORITHMS GROUP 1992. The NAG FORTRAN Library Manual, Mark 15. NAG Ltd, Oxford.
PICHOT, C. AND TEISSIER DU CROS, E. 1989. Estimation of genetic parameters in eastern cottonwood Populus deltoides Bartr. Consequence for the breeding strategy. Ann. Sci. For, 46, 307-324.

REBAï, A. AND GOFFINET, B. 1993. Power of tests for QTL detection using replicated progenies derived from a diallel cross. Theor. Appl. Genet., 86, 1014-1022.

REBAï, A., GOFFINET, B. AND MANGIN, B. 1995. Comparing power of different methods for QTL detection. Biometrics, 51, 87-99.

RODOLPHE, F. AND LEFORT, M. 1993. A multi-marker model for detecting chromosomal segments displaying QTL activity. Genetics, 134, 1277-1288.

SAX, K. 1923. The association of size differences with seed coat pattern and pigmentation in Phaseolus vulgaris. Genetics, 8, 552-560.

SOLLER, M. AND BECKMANN, J. S. 1990. Marker based mapping of quantitative trait loci using replicated progenies. Theor. Appl. Genet., 80, 205-208.

SOLLER, M. AND GENIZI, A. 1978. The efficiency of experimental designs for the detection of linkage between a marker locus and a locus affecting a quantitative trait in segregating populations. Biometrics, 34, 47-55.

SOLLER, M., GENIZI, A. AND BRODY, T. 1976. On the power of experimental designs for the detection of linkage between marker loci and quantitative loci in crosses between inbred lines. Theor. Appl. Genet., 47, 35-39.

STONECYPHER, R. W. AND McCULLOUGH, R. B. 1986. Estimates of additive and non-additive genetic variances from a clonal diallel of Douglas fir Pseudotsuga mensiesii Mirb. Franco. In: Proceedings IUFRO Joint meeting of working parties on breeding theory, progeny testing, and seed orchards, Williamsburg, VA, 13-17 October 1986, pp. 211-227.

TANKSLEY, S. D., MEDINA-FILHO, H. AND RICK, C. M. 1982. Use of naturally occurring enzyme variation to detect and map genes controlling quantitative traits in an interspecific backcross of tomato. Heredity, 49, 11-25.

VAN ECK, H. J., JACOBS, J. M., STAM, P., TON, J. AND STIEKEMA, w. J. 1994. Multiple alleles for tuber shape in diploid potato detected by qualitative and quantitative genetic analysis using RFLPs. Genetics, 137, 303-309.

WANG, G.-L., MACKILL, D. J., BONMAN, J. M., McCOUCH, S. R., CHAMPOUX, M. C. AND NELSON, R. J. 1994. RFLP mapping of genes conferring complete and partial resistance to blast in a durably resistant rice cultivar. Genetics, 136, 1421-1434.

WELLER, J. I. 1986. Maximum likelihood techniques for the mapping and analysis of quantitative trait loci with the aid of genetic markers. Biometrics, 42, 627-640.

WELLER, J. 1., KASHI, Y. AND SOLLER, M. 1990. Power of daughter and granddaughter designs for determining linkage between marker loci and quantitative trait loci in dairy cattle. J. Dairy Sci., 73, 2525-2537.

williams, C. G. AND NeAle, D. B. 1992. Conifer wood quality and marker-aided selection, a case study. Can. J. Forest Res., 22, 1009-1017.

ZENG, Z. B. 1994. Precision mapping of quantitative trait loci. Genetics, 136, 1457-1468. 


\section{Appendix}

To calculate the non-centrality parameter $\lambda$, we first observed that $\mathbf{K} \boldsymbol{\beta}=\boldsymbol{\beta}_{2}=\left[q_{i}\right]_{i=1, \ldots, p}$. Besides, with the constraint applied to gca parameters and the parameterization chosen, the $\mathbf{Z}_{0}, \mathbf{Z}_{1}$ and $\mathbf{Z}_{2}$ matrices of the design matrix $\mathbf{Z}$ are orthogonal, i.e. $\mathbf{Z}_{0}^{\prime} \mathbf{Z}_{1}=0$,

$\mathbf{Z}_{0}^{\prime} \mathbf{Z}_{2}=0$ and $\mathbf{Z}_{1}^{\prime} \mathbf{Z}_{2}=0$. As a consequence,

$\left(\mathbf{Z}^{\prime} \mathbf{Z}\right)^{-1}=\left[\begin{array}{lll}\left(\mathbf{Z}_{0}^{\prime} \mathbf{Z}_{0}\right)^{-1} & 0 & 0 \\ 0 & \left(\mathbf{Z}_{1}^{\prime} \mathbf{Z}_{1}\right)^{-1} & 0 \\ 0 & 0 & \left(\mathbf{Z}_{2}^{\prime} \mathbf{Z}_{2}\right)^{-1}\end{array}\right]$

and $\mathbf{K}\left(\mathbf{Z}^{\prime} \mathbf{Z}\right)^{-1} \mathbf{K}^{\prime}=\left[\left(\mathbf{Z}_{2}^{\prime} \mathbf{Z}_{2}\right)^{-1}\right]$.
Hence $\lambda=\left(\mathbf{Z}_{2} \boldsymbol{\beta}_{2}\right)^{\prime}\left(\mathbf{Z}_{2} \boldsymbol{\beta}_{2}\right) / 2 \sigma^{2}$. Thus the exact value of $\lambda$ depends on the number of offspring of each parent, and on a difference $\delta_{i j}$ between the numbers of individuals belonging to each genotypic class in each family (see Table 1). When the number of individuals per family is constant (this can be controlled), the expected number of offspring per parent is $2 N / p$ for diallel, cyclic and single-pair designs, and it is $N / p_{1}$ for the $p_{1}$ parents of the first group, and $N / p_{2}$ for the $p_{2}$ parents of the second group in a factorial design. Besides, the expected number of individuals per genotypic class in a family is constant because the four classes segregate in a $1: 1: 1: 1$ ratio. Consequently, the expected value of $\delta_{i j}$ is null. 